\title{
Noise Reduction on the LEP bunch current measurement system
}

\author{
A. Burns, B. Halvarsson, H. Jakob, H. Schmickler
}

\section{Abstract}

The lifetimes of coasting beams in LEP are computed from the difference of the bunch currents measured during a time interval of a few seconds. The quality of these measurements is presently limited by the noise figure of the bunch to bunch aquistion system. This paper decribes comparative studies on the aquistion system in order to improve the noise figure by one order of magnitude. For this purpose three different principles, i.e. integrating measurements, peak hold circuits with internal timing (autotrigger) or peak hold circuits with external timing were studied.

The paper describes the results of these studies.

\section{INTRODUCTION}

The LEP bunch current monitoring system has been described in previous publications [1] [2] [3]. The system is used for daily LEP operation and an online display of the measured bunch currents and computed beam lifetimes is available in the main control room. Fig.1 shows a screen dump of the online display for the positron bunches during a LEP physics coast with four bunchtrains of four bunches in each train. In addition to the bunch currents the lifetime averaged over all bunches is displayed (56.4 hours in fig. 1 ) and the time evolution of the lifetime is shown as the yellow trace. Although the beam conditions had not changed a significant variation of the measured lifetime is visible in the graph. This variation is linked to noise contributions in the individual current measurements and will be discussed in the following section.

Fig.2 illustrates how the lifetime is computed from two consecutive bunch current measurements, which themselves are averages of many beam revolutions during a time

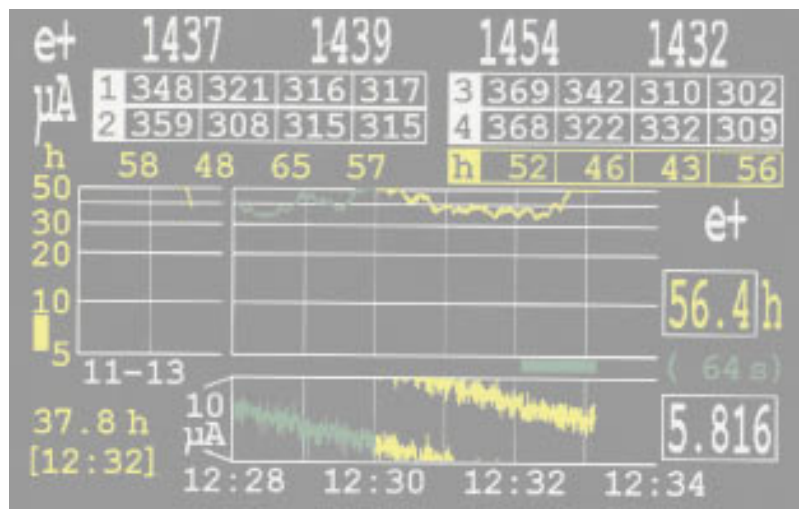

Figure 1: Online Display in the main control room of the LEP-BCT interval $\Delta \mathrm{t}$. Error propagation yields for the measurement error on the lifetimes:

$$
\frac{\sigma_{\tau}}{\tau}=\frac{\tau \cdot \sigma_{\Delta I}}{I \cdot \Delta t^{3 / 2}}
$$

where $\sigma_{\Delta I}$ is the variance of the measured current difference, which can be evaluated as $\sqrt{2}$ times the variance of indivudual current measurements. The above equation shows the strong influence of the integration time interval $\Delta t$. In the present LEP system this is exploited by having a dynamic adjustment of the integration time. For long lifetimes the integration time is increased up to 128 seconds, and only for short lifetimes integration times of 1 second are used (see details in [4]).

Of course a better solution to the problem would be to reduce drastically the variance of individual current measurements, such that the system could be operated with a fixed integration time of 1 or 2 seconds. This is very convinient for operators, as the recovery time of the instrument after sudden lifetime changes is much quicker.

Using equation 1 with an integration time of 2 seconds and asking for $5 \%$ of measurement error on lifetimes of about 20 hours requires a current measurement noise of about $20 \mathrm{ppm}$. This has to be compared with the value of the present BCT system of $100-200 \mathrm{ppm}$.

The following chapters describe measurement results on different acquistion systems in order to obtain the required factor 10 improvement in noise performance.

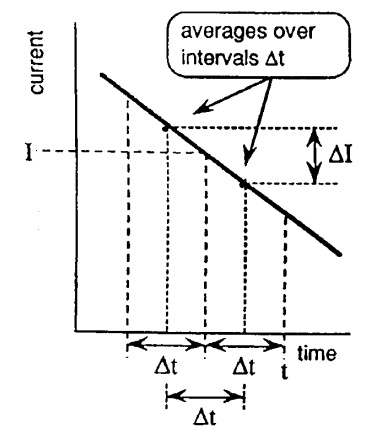

$$
\begin{aligned}
& \text { Exponential beam current } \\
& \text { decay with lifetime } \tau \text { : } \\
& \qquad \mathrm{I}=\mathrm{I}_{\mathrm{o}} \mathrm{e}^{-\mathrm{t} / \tau} \\
& \text { For } \Delta \mathrm{t}<<\tau \\
& \text { best estimate of lifetime } \\
& \text { in interval }(\mathrm{t}-2 \Delta \mathrm{t}, \mathrm{t}) \\
& \qquad \tau=\frac{\mathrm{I} \Delta \mathrm{t}}{\Delta \mathrm{I}} \\
& \text { Relative error in lifetime } \\
& \text { (for } \left.\sigma_{\Delta \mathrm{I}} / \Delta \mathrm{I}<<1\right) \\
& \qquad \frac{\sigma_{\tau}}{\tau}=\frac{\tau \sigma_{\Delta \mathrm{I}}}{\mathrm{I} \Delta \mathrm{t} / 2},
\end{aligned}
$$

Figure 2: Illustration of lifetime calculation from two consecutive beam current measurements 


\section{ACQUISITION ELECTRONICS}

\subsection{Signal Integration}

This circuit is used in the present BCT system and its design was aimed at obtaining maximum absolute precision. The bunch signal (1) is measured as difference between the integrated beam signal and an integration of the baseline (pedestal) of the detector. The subtraction is done as analog circuit. Two "Integrate and Hold" Circuits, which are controlled by two timing gates (2) \& (3) of equal length, are used for the integration.

\subsection{Peak Hold Circuits}

Compared to the previous circuit a peak hold circuit is not optimum in terms of absolute precision as the baseline of the signal is neglected, on the contrary the circuit can be optimized for best noise performance. The signal to noise ratio depends largely on the bandwidth of the input signal and the jitter on the sampling gate. In bunch train operation the spacing between two bunches of the same polarity is $335 \mathrm{nsec}$. In order to have less than $1 \%$ crosstalk between bunches the transformer signal was filtered with a lowpass of $12 \mathrm{MHz}$ corner frequency. The noise requirements of 20 ppm translate into the specification of having a jitter on the sampling gate of less than $3 \mathrm{nsec}$ rms.

Two circuits with a different circuit for the sampling gate were tested. All tests were done by using modified digitizing cards of the BOSC system [5], which is presently used for the SPS tune measurements.

\subsubsection{Internal timing}

The objective of this design was to render the system almost independent of the LEP filling scheme as the sampling gate is derived from the beam signal itself. The signal is split into a direct path (1) and into a delayed pass (2). A comparator circuit detects the cross over between direct and delayed signal. By adjusting the amplitude of the delayed signal the cross over point can be set such that the end of the sampling gate coincides with the crest of the input signal at the $\mathrm{S} \& \mathrm{H}$ circuit.

\subsubsection{External Timing}

In this case the "end of sampling gate" is applied from the outside to the circuit. For the tests a signal directly derived from the Rf-system was used. This signal had a timing jitter below $3 \mathrm{nsec}$ rms.

\section{RESULTS}

Fig.4 shows measurements of the the bunch currents using the three different electronics as described in the previous chapter. Each point in the graphs corresponds to the average of 5622 machine turns, i.e. the equivalent of $0.5 \mathrm{sec}-$ onds of data.
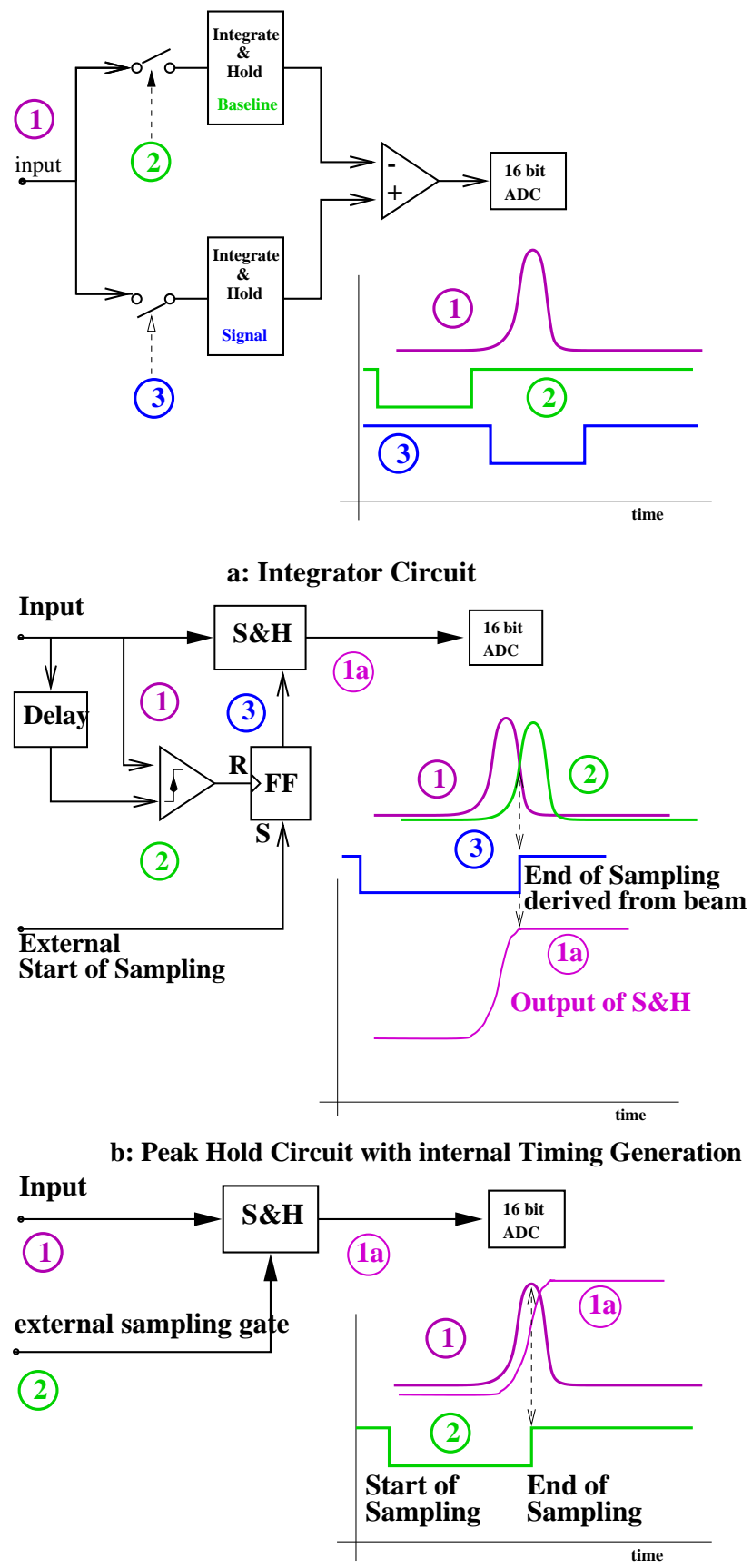

c: Peak Hold Circuit with external Timing Generation

Figure 3: Block Diagram and Timing Sequence of the three different electronics circuits, top: integrator, middle: peak hold with internal timing, bottom: peak hold with external timing 
A straight line fit is applied to the data and the rms spread around the fitted curve is displayed in the measurement graph. The obtained rms values depend on the selection of the input data, as the straight line fit implies a constant life time during the measurements. With different data sets the following range for the noise figures were obtained: integrator: 120 - $300 \mathrm{ppm}$, peak hold internal timing: 80 $140 \mathrm{ppm}$, peak hold external timing: $19-37 \mathrm{ppm}$.

\section{DISCUSSION}

The above results show clearly that both peak hold circuits give better noise performance than the present integrator circuit. Since the prototype design in 1989 the present BCT system has the integrator circuits and the digitizing electronics in separate chassis making it very difficult to avoid ground loops or other noise sources. A modern and more compact circuit design would probably give also better results.

The choice between the two peak hold circuits is almost evident. Only the external timing circuit yields the required performance in terms of rms noise. Another argument in favour of the external timing circuit is the required dynamic range. LEP is filled with bunches between $1 \mu \mathrm{A}$ bunch current in special machine experiments up to $1 \mathrm{~mA}$ bunch currents in high intensity operation. In case of an internal autotrigger circuit this dynamic range of $60 \mathrm{~dB}$ can not be realized without switching the gain of an preamplifier. The best result for the dynamic range of the autotrigger, which we achieved in the laboratory was $42 \mathrm{~dB}$ (for the bandwidth considerations as described in the previous chapter).

So in conclusion the new LEP BCT system scheduled for 1997 operation will make use of peak hold circuits with external timing reference. With a short integration window of 2 seconds on the bunch current measurements (see fig.1) and $20 \mathrm{ppm}$ rms noise lifetimes of 20 hours will be measured with a precision of $5 \% \mathrm{rms}$.

\section{REFERENCES}

[1] K.B.Unser, Measuring bunch intensity, beam loss and bunch lifetime in LEP",

Proceedings of the 2nd European Particle Accelerator Conference, Nice, France, June 1990, pp. 786-788.

[2] G.Burtin et al., "Mechanical Design, Signal Processing and Operator Interface of the LEP beam current transformers", Proceedings of the 2nd European Particle Accelerator Conference, Nice, France, June 1990, pp. 794-796.

[3] L. Vos, "The LEP monitor for the measurement of bunch intensity", CERN SL/94-18 (BI), May 1994.

[4] A.J.Burns et al.,"Real Time Monitoring of LEP Beam Currents and Lifetimes

Proceedings of the 4th European Particle Accelerator Conference, London, Great Britain, June 1994, pp. 1716-1718.

[5] A.J. Burns et al., "The BOSC project", CERN Divisional Note, SL 90-68(AP)
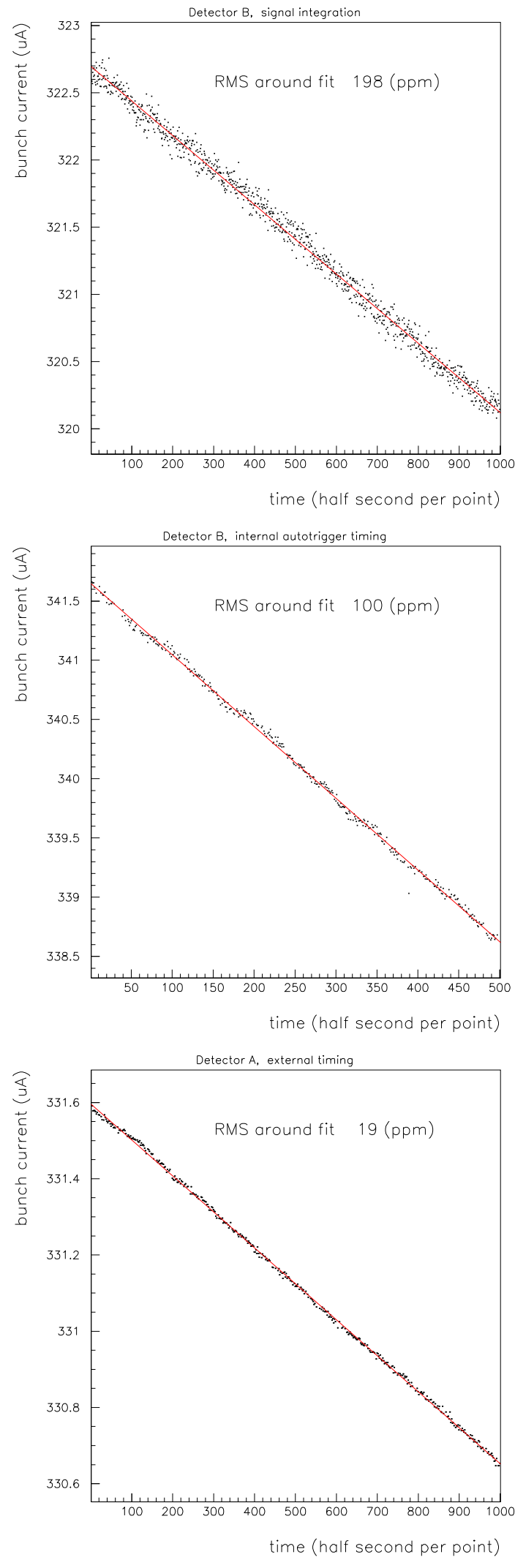

Figure 4: Noise measurements on the three different electronic circuits: top: integrator, middle: peak hold with internal timing, bottom: peak hold with external timing reference 